\title{
Drawdowns preceding rallies in the Brownian motion model
}

\author{
OLYMPIA HADJILIADIS $\uparrow$ and JAN VEČER $\breve{R}^{*}+$ \\ $\dagger$ Department of Mathematics, Drexel University, \\ Philadelphia, PA 19104, USA \\ tDepartment of Statistics, Columbia University, \\ New York, NY 10027, USA
}

(Received 4 January 2006; in final form 18 April 2006)

\begin{abstract}
We study drawdowns and rallies of Brownian motion. A rally is defined as the difference of the present value of the Brownian motion and its historical minimum, while the drawdown is defined as the difference of the historical maximum and its present value. This paper determines the probability that a drawdown of $a$ units precedes a rally of $b$ units. We apply this result to examine stock market crashes and rallies in the geometric Brownian motion model.
\end{abstract}

Keywords: Drawdowns; Brownian motion model; Rallies

\section{Introduction}

In this paper we determine the probability that a drawdown precedes a rally in the Brownian motion model. The probabilities are computed by means of the distribution function of the random variables $y_{T_{1}(a)}^{+}$and $y_{T_{2}(b)}^{-}$, where $y_{T_{1}(a)}^{+}$represents the value of the upward rally when the drawdown process reaches the level $a$ for the first time, and $y_{T_{2}(b)}^{-}$represents the value of the drawdown when the upward rally process reaches the level $b$ for the first time. Using the results of Taylor (1975) and Lehoczky (1977) concerning the distribution of a stopped drifted Brownian motion at the first time of the downfall of level $a$, we are able to show that the probability density functions of $y_{T_{1}(a)}^{+}$and $y_{T_{2}(b)}^{-}$are exponential, but with a discrete mass at 0 . The paper concludes with a solution to the problem of computing the probability that a drop of $(100 \cdot \alpha) \%$ from the running maximum of a stock price occurs before a rally of $(100 \cdot \beta) \%$ from its running minimum, given that the stock price follows a geometric Brownian motion.

Risk management of drawdowns and portfolio optimization with drawdown constraints is becoming increasingly important among practitioners. Chekhlov et al. (2005) studied drawdown measures in portfolio optimization. Magdon-Ismail et al. (2004) determined

*Corresponding author. Email: vecer@stat.columbia.edu the distribution of the maximum drawdown of Brownian motion.

Our results are related to a recent paper by Meijilson (2003), where the results of Taylor (1975) and Lehoczky (1977) are used to derive the expected time to a given drawdown of Brownian motion, as well as the stationary distribution of the drawdown process. An alternative derivation of the above expected value based on the expected delay of the CUSUM stopping time appears in Hadjiliadis and Moustakides (2005). The CUSUM stopping time was first proposed by Page (1954) and was used subsequently as a means of detecting a regime change in the Brownian motion model (Beibel 1996, Shiryaev 1996, Moustakides 2004).

In the next section we first outline the main results of the paper. We then proceed to give an example of an application of these results in the examination of stock market crashes and rallies. We finally give some concluding remarks.

\section{Results}

Consider the process $X_{t}$ with the following dynamics:

$$
X_{t}=\sigma W_{t}+\gamma t,
$$

where $\gamma, \sigma \in \mathbb{R}$ and $W_{t}$ is a standard Brownian motion process. 
The quantity

$$
X_{t}-\inf _{s \in[0, t]} X_{s}
$$

measures the size of the rally, comparing the present value of the process with its historical minimum, while the quantity

$$
\sup _{s \in[0, t]} X_{s}-X_{t}
$$

measures the size of the drawdown, comparing the present value of the process with its historical maximum.

The aim of this section is to determine the probability that a drawdown of size $a$ precedes a rally of size $b$. We introduce the stopping times:

$$
T_{1}(a)=\inf \left\{t \geq 0: \sup _{s \in[0, t]} X_{s}-X_{t}=a, a \in \mathbb{R}_{+}\right\},
$$

and

$$
T_{2}(b)=\inf \left\{t \geq 0: X_{t}-\inf _{s \in[0, t]} X_{s}=b, b \in \mathbb{R}_{+}\right\} .
$$

Consider the stopping time $T(a, b)=T_{1}(a) \wedge T_{2}(b)$. The stopping times $T_{1}(a)$ and $T_{2}(b)$ indicate, respectively, the first time that the drawdown process reaches the critical level $a, T_{1}(a)$, and the first time the upward rally process reaches the critical level $b, T_{2}(b)$. In this section, we compute the probabilities of the event $\{T(a, b)=$ $\left.T_{1}(a)\right\}$, which represents the event that a drawdown of size $a$ occurs before the rally of size $b$, and $\left\{T(a, b)=T_{2}(b)\right\}$, which represents the event that a rally of size $b$ occurs before the drawdown of size $a$.

In order to simplify notation we introduce the following processes:

$$
\begin{aligned}
m_{t}^{+} & :=\inf _{s \in[0, t]} X_{s}, \\
m_{t}^{-} & :=\inf _{s \in[0, t]}\left(-X_{s}\right)=-\sup _{s \in[0, t]} X_{s}, \\
y_{t}^{+} & :=X_{t}-m_{t}^{+}, \\
y_{t}^{-} & :=-X_{t}-m_{t}^{-} .
\end{aligned}
$$

Using the above notation, the stopping times $T_{1}(a)$ and $T_{2}(b)$ become

$$
\begin{aligned}
& T_{1}(a)=\inf \left\{t \geq 0: y_{t}^{-}=a, a \in \mathbb{R}_{+}\right\} \\
& T_{2}(b)=\inf \left\{t \geq 0: y_{t}^{+}=b, b \in \mathbb{R}_{+}\right\}
\end{aligned}
$$

Theorem 2.1: Let $X_{t}=\sigma W_{t}+\gamma t$ be a standard Brownian motion with drift parameter $\gamma \in \mathbb{R}$, and variance parameter $\sigma \in \mathbb{R} ; T, T_{1}$ and $T_{2}$ are the stopping times defined above. We distinguish the following two cases:

(1) $b \geq a>0$

The probability of the drawdown preceding the rally, or the rally preceding the drawdown, is given, respectively, by

$$
\begin{aligned}
P\left(T(a, b)=T_{1}(a)\right)= & m_{A}+\left(1-m_{A}\right) \\
& \times\left[1-\exp \left(-\frac{2 \gamma / \sigma^{2}}{e^{\left(2 \gamma / \sigma^{2}\right) a}-1} \cdot(b-a)\right)\right],
\end{aligned}
$$

$P\left(T(a, b)=T_{2}(b)\right)=\left(1-m_{A}\right) \cdot \exp \left(-\frac{2 \gamma / \sigma^{2}}{e^{\left(2 \gamma / \sigma^{2}\right) a}-1} \cdot(b-a)\right)$,

where

$$
m_{A}=\frac{e^{-\left(2 \gamma / \sigma^{2}\right) a}+\left(2 \gamma / \sigma^{2}\right) a-1}{e^{\left(2 \gamma / \sigma^{2}\right) a}+e^{-\left(2 \gamma / \sigma^{2}\right) a}-2} .
$$

(2) $a \geq b>0$

The probability of the drawdown preceding the rally, or the rally preceding the drawdown, is given, respectively, by

$$
\begin{aligned}
P\left(T(a, b)=T_{1}(a)\right)= & \left(1-m_{B}\right) \\
& \times \exp \left(-\frac{2 \gamma / \sigma^{2}}{1-e^{-\left(2 \gamma / \sigma^{2}\right) b}} \cdot(a-b)\right), \\
P\left(T(a, b)=T_{2}(b)\right)= & m_{B}+\left(1-m_{B}\right) \\
& \times\left[1-\exp \left(-\frac{2 \gamma / \sigma^{2}}{1-e^{-\left(2 \gamma / \sigma^{2}\right) b}} \cdot(a-b)\right)\right],
\end{aligned}
$$

where

$$
m_{B}=\frac{e^{\left(2 \gamma / \sigma^{2}\right) b}-\left(2 \gamma / \sigma^{2}\right) b-1}{e^{\left(2 \gamma / \sigma^{2}\right) b}+e^{-\left(2 \gamma / \sigma^{2}\right) b}-2} .
$$

The proof of the theorem uses the following proposition.

Proposition 2.2: The probability distribution functions of the random variables $y_{T_{1}(a)}^{+}$and $y_{T_{2}(b)}^{-}$are given by

(1) $P\left(y_{T_{1}(a)}^{+}=0\right)=m_{A}$,

$$
\begin{aligned}
P\left(y_{T_{1}(a)}^{+} \in \mathrm{d} r\right)= & \left(1-m_{A}\right) \cdot\left[\frac{2 \gamma / \sigma^{2}}{e^{\left(2 \gamma / \sigma^{2}\right) a}-1}\right. \\
& \left.\times \exp \left(-\frac{2 \gamma / \sigma^{2}}{e^{\left(2 \gamma / \sigma^{2}\right) a}-1} \cdot r\right)\right] \mathrm{d} r, \quad r>0,
\end{aligned}
$$

where $m_{A}$ is given by equation (3).

(2) $P\left(y_{T_{2}(b)}^{-}=0\right)=m_{B}$,

$$
\begin{aligned}
P\left(y_{T_{2}(b)}^{-} \in \mathrm{d} r\right)= & \left(1-m_{B}\right) \cdot\left[\frac{2 \gamma / \sigma^{2}}{1-e^{-\left(2 \gamma / \sigma^{2}\right) b}}\right. \\
& \left.\times \exp \left(-\frac{2 \gamma / \sigma^{2}}{1-e^{-\left(2 \gamma / \sigma^{2}\right) b}} \cdot r\right)\right] \mathrm{d} r, \quad r>0,
\end{aligned}
$$

where $m_{B}$ is given by equation (6). 

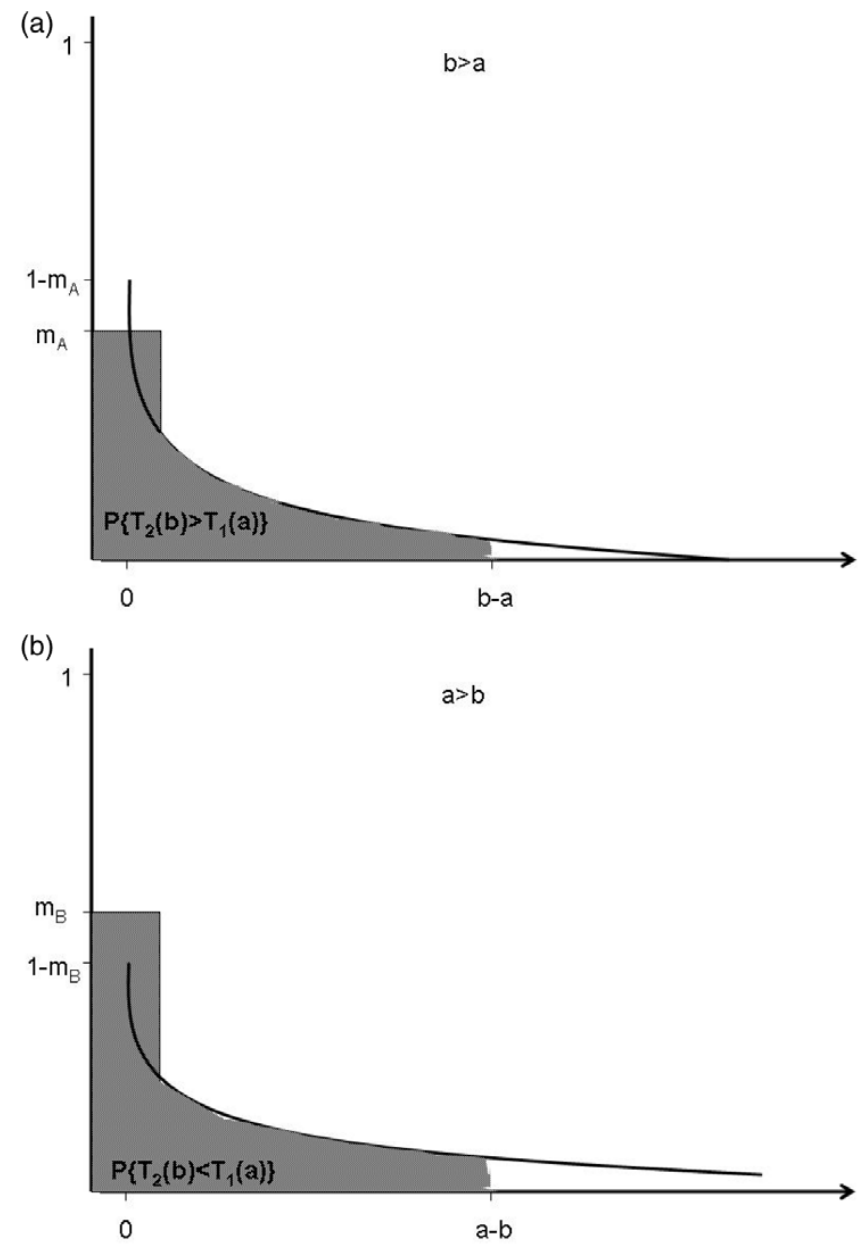

Figure 1. (a) Distribution of the random variable $y_{T_{1}(a)}^{+}$, and the gray area marks $P\left(T(a, b)=T_{1}(a)\right)$, in the case where $b \geq a$. (b) Distribution of the random variable $y_{T_{2}(b)}^{-}$, and the gray area marks $P\left(T(a, b)=T_{2}(b)\right)$, in the case where $a \geq b$.

Before we proceed to the proof of the two above results, let us notice that all of equations (1)-(6), as well as the distributions of the random variables $y_{T_{1}(a)}^{+}$and $y_{T_{2}(b)}$, as they appear in Proposition 2.2, depend on the ratio $2 \gamma / \sigma^{2}$. This ratio is called the adjustment coefficient and appears in the insurance risk literature (Asmussen 2000). To illustrate the probabilities that appear in Theorem 2.1, we include a graph of the densities of the random variables $y_{T_{1}(a)}^{+}$and $y_{T_{2}(b)}^{-}$(see figure 1 ).

In order to prove Proposition 2.2 and Theorem 2.1, we will need the following two lemmas.

Lemma 2.3: For $a, b \in \mathbb{R}_{+}$, we have

$$
\begin{aligned}
& E\left[T_{1}(a)\right]=\frac{e^{\left(2 \gamma / \sigma^{2}\right) a}-\left(2 \gamma / \sigma^{2}\right) a-1}{\left(2 \gamma / \sigma^{2}\right)^{2}}, \\
& E\left[T_{2}(b)\right]=\frac{e^{-\left(2 \gamma / \sigma^{2}\right) b}+\left(2 \gamma / \sigma^{2}\right) b-1}{\left(2 \gamma / \sigma^{2}\right)^{2}} .
\end{aligned}
$$

Proof: Let $g(x)=e^{-\left(2 \gamma / \sigma^{2}\right) x}+\left(2 \gamma / \sigma^{2}\right) x-1$. By applying Itô's rule to the process $g\left(y_{t}^{+}\right)$we get

$$
\begin{aligned}
\mathrm{d} g\left(y_{t}^{+}\right)= & \sigma g^{\prime}\left(y_{t}^{+}\right) \mathrm{d} W_{t}+\gamma g^{\prime}\left(y_{t}^{+}\right) \mathrm{d} t-g^{\prime}\left(y_{t}^{+}\right) \mathrm{d} m_{t}^{+} \\
& +\frac{1}{2} \sigma^{2} g^{\prime \prime}\left(y_{t}^{+}\right) \mathrm{d} t .
\end{aligned}
$$

We notice that the third term on the right-hand side of the above equality disappears because $d m_{t}^{+} \neq 0$ only when $y_{t}^{+}=0$ and $g^{\prime}(0)=0$. We also notice that the function $g$ satisfies the second-order differential equation

$$
\gamma g^{\prime}(x)+\frac{1}{2} \sigma^{2} g^{\prime \prime}(x)=\frac{2 \gamma^{2}}{\sigma^{2}} .
$$

Solving equation (13), we get

$$
g\left(y_{t}^{+}\right)-g(0)=\int_{0}^{t} \sigma g^{\prime}\left(y_{s}^{+}\right) \mathrm{d} W_{s}+\int_{0}^{t}\left(\gamma g^{\prime}\left(y_{t}^{+}\right)+\frac{1}{2} g^{\prime \prime}\left(y_{t}^{+}\right)\right) \mathrm{d} s .
$$

Let

$$
S_{n}=\inf \left\{t \geq 0 ; \frac{2 \gamma^{2}}{\sigma^{2}} t \geq n\right\} .
$$

Let $T_{2}^{n}(b)=T_{2}(b) \wedge S_{n}$. Obviously, $T_{2}^{n}(b)$ is a.s. finite. On the event $\left\{T_{2}^{n}(b) \geq t\right\}$, we have $\left\{y_{t}^{+} \leq b\right\}$. Consequently,

$$
E\left[\int_{0}^{T_{2}^{n}(b)} \frac{2 \gamma^{2}}{\sigma^{2}} g^{\prime}\left(y_{s}^{+}\right) \mathrm{d} s\right] \leq\left(g^{\prime}(b)\right)^{2} n<\infty .
$$

Evaluating (15) at $T_{2}^{n}(b)$, and taking expectations while using equations (14) and (16), we get

$$
E\left[g\left(y_{T_{2}^{n}(b)}^{+}\right)\right]=\frac{2 \gamma^{2}}{\sigma^{2}} E\left[T_{2}^{n}(b)\right] .
$$

But

$$
g(b) \geq E\left[g\left(y_{T_{2}^{n}(b)}^{+}\right)\right] .
$$

Hence we have that

$$
g(b) \geq \frac{2 \gamma^{2}}{\sigma^{2}} E\left[T_{2}^{n}(b)\right] \geq \frac{2 \gamma^{2}}{\sigma^{2}} E\left[1_{\left\{T_{2}^{n}=\infty\right\}}\right] .
$$

Letting $n \rightarrow \infty$ and using the bounded convergence theorem, we deduce that $T_{2}(b)$ is finite a.s. as well.

Evaluating both sides of equation (15) at $T_{2}(b)$, and taking expectations of both sides, while using equation (14), and the fact that the quadratic variation of the stochastic integral $\int_{0}^{T_{2}(b)} g^{\prime}\left(y_{t}^{+}\right) \mathrm{d} W_{t}$ is finite (this follows from equation (16) and the a.s. finiteness of $\left.T_{2}(b)\right)$, we get

$$
g(b)=\frac{2 \gamma^{2}}{\sigma^{2}} E\left[T_{2}(b)\right] .
$$

Consequently,

$$
E\left[T_{2}(b)\right]=\frac{g(b)}{2 \gamma^{2} / \sigma^{2}} .
$$

Similarly, we can show that

$$
E\left[T_{1}(a)\right]=\frac{g(-a)}{\left(2 \gamma / \sigma^{2}\right)^{2}} .
$$

This concludes the proof of the lemma. 
Lemma 2.4: We have

$$
y_{t}^{+}+y_{t}^{-}=\max \left\{\sup _{s \leq t} y_{s}^{+}, \sup _{s \leq t} y_{s}^{-}\right\} .
$$

Proof: Observe that

$$
y_{t}^{+}+y_{t}^{-}=-m_{t}^{+}-m_{t}^{-} \text {. }
$$

We notice that the process $y_{t}^{+}+y_{t}^{-}$can only increase when either $X_{t}=m_{t}^{+}$or $-X_{t}=m_{t}^{-}$, both of which cannot happen at the same time, since that would imply that $y_{t}^{+}+y_{t}^{-}$is 0 . Therefore, $y_{t}^{+}+y_{t}^{-}$is a constant as a function of time unless either $y_{t}^{+}=0$ or $y_{t}^{-}=0$, at which instant $t$, we simultaneously have

(1) $\max \left\{y_{t}^{+}, y_{t}^{-}\right\}=\max \left\{\left\{\sup _{s \leq t} y_{s}^{+}, \sup _{s \leq t} y_{s}^{-}\right\}\right\}$,

(2) $\sup _{s \leq t}\left(y_{s}^{+}+y_{s}^{-}\right)=y_{t}^{+}+y_{t}^{-}$.

This completes the proof of the lemma.

As a consequence of this lemma we have

$$
\begin{aligned}
& y_{T_{1}(a)}^{+}=\left(\max _{t \leq T_{1}(a)} y_{t}^{+}-a\right) \vee 0, \\
& y_{T_{2}(b)}^{-}=\left(\max _{t \leq T_{2}(b)} y_{t}^{-}-b\right) \vee 0 .
\end{aligned}
$$

Finally, in order to proceed to the proof of Proposition 2.2 and Theorem 2.1, we will use the results of Taylor (1975) and Lehoczky (1977). Taylor computes the bivariate Laplace transform of $X_{T_{1}(a)}$ and $T_{1}(a)$, where $T_{1}$ is defined as above. Lehoczky pointed out that the random variable $X_{T_{1}(a)}+a=\sup _{t \leq T_{1}(a)} X_{t}$ has the exponential distribution

$$
X_{T_{1}(a)}+a \sim \exp \left(\frac{2 \gamma / \sigma^{2}}{e^{\left(2 \gamma / \sigma^{2}\right) a}-1}\right) .
$$

Note that the exponential parameter becomes equal to $1 / a$ in the case when $\gamma=0$. Now we can proceed to the proof of Proposition 2.2 and then to the proof of Theorem 2.1.

Proof (Proof of Proposition 2.2): We will only compute the probability density function of the random variable $y_{T_{1}(a)}^{+}$since the computation of the probability density function of the random variable $y_{T_{2}(b)}^{-}$is done in a similar way. From equation (22), it follows that

$$
P\left(y_{T_{1}(a)}^{+}=0\right)=P\left(\max _{t \leq T_{1}(a)} y_{t}^{+}<a\right),
$$

while

$$
\begin{aligned}
P\left(y_{T_{1}(a)}^{+} \in \mathrm{d} r\right)= & P\left(\max _{t \leq T_{1}(a)} y_{t}^{+} \geq a\right) \\
& \times P\left(y_{T_{1}(a)}^{+} \in \mathrm{d} r \mid \max _{t \leq T_{1}(a)} y_{t}^{+} \geq a\right) \\
= & P\left(y_{T_{1}(a)}^{+}>0\right) \cdot P\left(y_{T_{1}(a)}^{+} \in \mathrm{d} r \mid y_{T_{1}(a)}^{+}>0\right), \\
& r>0 .
\end{aligned}
$$

In the following discussion we first demonstrate

$$
\mathcal{L}\left(y_{T_{1}(a)}^{+} \mid y_{T_{1}(a)}^{+}>0\right)=\mathcal{L}\left(X_{T_{1}(a)}+a\right) .
$$

To see this, let

$$
R_{1}=\sup \left\{t \leq T_{1}(a) ; y_{t}^{+}=0\right\} .
$$

Fix $r>0$. Then

$$
\begin{aligned}
P( & \left.y_{T_{1}(a)}^{+} \in \mathrm{d} r \mid y_{T_{1}(a)}^{+}>0\right) \\
= & \frac{P\left(X_{T_{1}(a)}-\inf _{s \leq T_{1}(a)} X_{s} \in \mathrm{d} r\right)}{P\left(\max _{t \leq T_{1}(a)} y_{t}^{+} \geq a\right)} \\
= & \frac{P\left(X_{T_{1}(a)}-X_{R_{1}} \in \mathrm{d} r \mid R_{1}<T_{1}(a)\right)}{P\left(\max _{t \leq T_{1}(a)} X_{t}-X_{R_{1}} \geq a \mid R_{1}<T_{1}(a)\right)} \\
= & \frac{P\left(X_{T_{1}(a)}-X_{R_{1}} \in \mathrm{d} r \mid R_{1}<T_{1}(a)\right)}{P\left(\max _{R_{1} \leq t \leq T_{1}(a)} X_{t}-X_{R_{1}} \geq a \mid R_{1}<T_{1}(a)\right)} \\
= & \frac{P\left(X_{T_{1}(a)} \in \mathrm{d} r\right)}{P\left(\max _{t \leq T_{1}(a)} X_{t} \geq a\right)} \\
= & \frac{\lambda e^{-\lambda r} e^{-\lambda a} \mathrm{~d} r}{e^{-\lambda a}}=\lambda e^{-\lambda r} \mathrm{~d} r=P\left(X_{T_{1}(a)}+a \in \mathrm{d} r\right),
\end{aligned}
$$

where

$$
\lambda=\frac{2 \gamma / \sigma^{2}}{1-e^{-\left(2 \gamma / \sigma^{2}\right) a}} .
$$

Note that the third equality holds because conditional on $R_{1}<T_{1}(a), \inf _{s \leq T_{1}(a)} X_{s}=\inf _{s \leq R_{1}} X_{s}$, and $\operatorname{mak}_{t \leq T_{1}(a)} X_{t}=$ $\max _{R_{1} \leq t \leq T_{1}(a)} \bar{X}_{t}$.

Therefore, we get

$$
P\left(y_{T_{1}(a)}^{+} \in \mathrm{d} r \mid y_{T_{1}(a)}^{+}>0\right) \sim \exp \left(\frac{2 \gamma / \sigma^{2}}{e^{\left(2 \gamma / \sigma^{2}\right) a}-1}\right), \quad r>0 .
$$

From equation (22), it follows that

$$
P\left(y_{T_{1}(a)}^{+}=0\right)=P\left(T_{1}(a)<T_{2}(a)\right) .
$$

To compute $P\left(T_{1}(a)<T_{2}(a)\right)$, we first notice that

$$
\begin{aligned}
& T_{1}(a)=T(a, b)+\left(T_{1}(a)-T(a, b)\right) \mathbf{1}_{\left\{T(a, b)=T_{2}(b)\right\}}, \\
& T_{2}(b)=T(a, b)+\left(T_{2}(b)-T(a, b)\right) \mathbf{1}_{\left\{T(a, b)=T_{1}(a)\right\}} .
\end{aligned}
$$

Taking expectations we get

$$
\begin{aligned}
& E\left[T_{1}(a)\right]=E[T(a, b)]+E\left[\left(T_{1}(a)-T(a, b)\right) \mathbf{1}_{\left\{T(a, b)=T_{2}(b)\right\}}\right] \\
& E\left[T_{2}(b)\right]=E[T(a, b)]+E\left[\left(T_{2}(b)-T(a, b)\right) \mathbf{1}_{\left\{T(a, b)=T_{1}(a)\right\}}\right] .
\end{aligned}
$$

With $a=b$ and equation (22), it follows that

$$
\begin{aligned}
& E\left[T_{1}(a)\right]=E[T(a, a)]+E\left[T_{1}(a)\right] \cdot P\left(T_{2}(a)<T_{1}(a)\right), \\
& E\left[T_{2}(a)\right]=E[T(a, a)]+E\left[T_{2}(a)\right] \cdot P\left(T_{1}(a)<T_{2}(a)\right) .
\end{aligned}
$$

Using

$$
P\left(T_{1}(a)<T_{2}(a)\right)+P\left(T_{2}(a)<T_{1}(a)\right)=1
$$


and equations (35) and (36), we conclude that

$$
P\left(T_{1}(a)<T_{2}(a)\right)=\frac{E\left[T_{2}(a)\right]}{E\left[T_{2}(a)\right]+E\left[T_{1}(a)\right]} .
$$

The result now follows by substituting (29), (30) and (37) into equations (26) and (25), while using Lemma 2.3. This completes the proof of Proposition 2.2.

Proof (Proof of Theorem 2.1): We will prove the theorem in the case that $b \geq a$ since the proof is similar in the case $a \geq b$. Suppose that $b \geq a$.

From Lemma 2.4 and equation (22), it follows that, on the event $\left\{T_{1}(a)<T_{2}(b)\right\}$, we have

$$
y_{T_{1}(a)}^{+}= \begin{cases}0, & \text { if } \max _{s \leq T_{1}(a)} y_{s}^{+}<a, \\ \max _{s \leq T_{1}(a)} y_{s}^{+}-a, & \text { if } a \leq \max _{s \leq T_{1}(a)} y_{s}^{+}<b .\end{cases}
$$

Therefore,

$$
P\left(T_{1}(a)<T_{2}(b)\right)=P\left(y_{T_{1}(a)}^{+}=0\right)+\int_{0^{+}}^{b-a} P\left(y_{T_{1}(a)}^{+} \in \mathrm{d} r\right)
$$

and the result is obtained from Proposition 2.2. This completes the proof of Theorem 2.1.

Corollary 2.5: Let $X_{t}=W_{t}$ be a standard Brownian motion and let $T, T_{1}$ and $T_{2}$ be stopping times defined as above. We distinguish the following two cases

(1) $b \geq a>0$

The probability of the drawdown preceding the rally, or the rally preceding the drawdown, is given, respectively, by

$$
\begin{aligned}
& P\left(T(a, b)=T_{1}(a)\right)=\frac{1}{2}+\frac{1}{2} \cdot\left[1-e^{-(1 / a)(b-a)}\right], \\
& P\left(T(a, b)=T_{2}(b)\right)=\frac{1}{2} \cdot e^{-(1 / a)(b-a)}
\end{aligned}
$$

(2) $a \geq b>0$

The probability of the drawdown preceding the rally, or the rally preceding the drawdown, is given, respectively, by

$$
\begin{aligned}
& P\left(T(a, b)=T_{1}(a)\right)=\frac{1}{2} \cdot e^{-(1 / b)(a-b)}, \\
& P\left(T(a, b)=T_{2}(b)\right)=\frac{1}{2}+\frac{1}{2} \cdot\left[1-e^{-(1 / b)(a-b)}\right] .
\end{aligned}
$$

Proof: It is a simple consequence of Theorem 2.1 by taking the limit as $\gamma \rightarrow 0$.

Corollary 2.6: Let $X_{t}=W_{t}$ be a standard Brownian motion. The probability distribution function of the random variables $y_{T_{1}(a)}^{+}$and $y_{T_{2}(b)}^{-}$are given by

$$
\text { (1) } \begin{aligned}
P\left(y_{T_{1}(a)}^{+}=0\right) & =\frac{1}{2}, \\
P\left(y_{T_{1}(a)}^{+} \in \mathrm{d} r\right) & =\frac{1}{2} \cdot\left[\frac{1}{a} e^{-(1 / a) r}\right] \mathrm{d} r, \quad r>0 .
\end{aligned}
$$

$$
\text { (2) } \begin{aligned}
P\left(y_{T_{2}(b)}^{-}=0\right) & =\frac{1}{2}, \\
P\left(y_{T_{2}(b)}^{-} \in \mathrm{d} r\right) & =\frac{1}{2} \cdot\left[\frac{1}{b} e^{-(1 / b) r}\right] \mathrm{d} r, \quad r>0 .
\end{aligned}
$$

Proof: The above corollary is a consequence of Proposition 2.2 by letting $\gamma / \sigma^{2} \rightarrow 0$.

A graph of the distribution of each of the random variables $y_{T_{1}(a)}^{+}$and $y_{T_{2}(b)}^{-}$is shown in figure 2 .

Example 2.7 (Stock market crashes and rallies): Suppose that we have a stock $S_{t}$ whose dynamics follow a geometric Brownian motion:

$$
\mathrm{d} S_{t}=\mu S_{t} \mathrm{~d} t+\sigma S_{t} \mathrm{~d} W_{t}
$$

What is the probability that this stock would drop by $(100 \cdot \alpha) \%$ before it incurs a rally of $(100 \cdot \beta) \%$ ? We can solve this problem by using Theorem 2.1. First observe that

$$
S_{t}=S_{0} \exp \left(\left(\mu-\frac{1}{2} \sigma^{2}\right) t+\sigma W_{t}\right)=S_{0} \exp \left(\sigma X_{t}\right)
$$

where

$$
X_{t}=W_{t}+\left(\frac{\mu}{\sigma}-\frac{\sigma}{2}\right) t
$$

Let $U_{1}(\alpha)$ be the first time the stock drops by $(100 \cdot \alpha) \%$ from its running maximum and $U_{2}(\beta)$ be the first time the stock rallies by $(100 \cdot \beta) \%$ from its running minimum. Let

$$
M_{t}=\sup _{0 \leq s \leq t} S_{s}
$$

and

$$
N_{t}=\inf _{0 \leq s \leq t} S_{s}
$$

Notice that

$$
\begin{aligned}
U_{1}(\alpha) & =\inf \left\{t \geq 0: S_{t} \leq(1-\alpha) M_{t}\right\} \\
& =\inf \left\{t \geq 0: \sup _{0 \leq s \leq t} X_{s}-X_{t} \geq-\frac{1}{\sigma} \log (1-\alpha)\right\} \\
& =T_{1}\left(-\frac{1}{\sigma} \log (1-\alpha)\right),
\end{aligned}
$$

and

$$
\begin{aligned}
U_{2}(\beta) & =\inf \left\{t \geq 0: S_{t} \geq(1+\beta) N_{t}\right\} \\
& =\inf \left\{t \geq 0: X_{t}-\inf _{0 \leq s \leq t} X_{s} \geq \frac{1}{\sigma} \log (1+\beta)\right\} \\
& =T_{2}\left(\frac{1}{\sigma} \log (1+\beta)\right)
\end{aligned}
$$



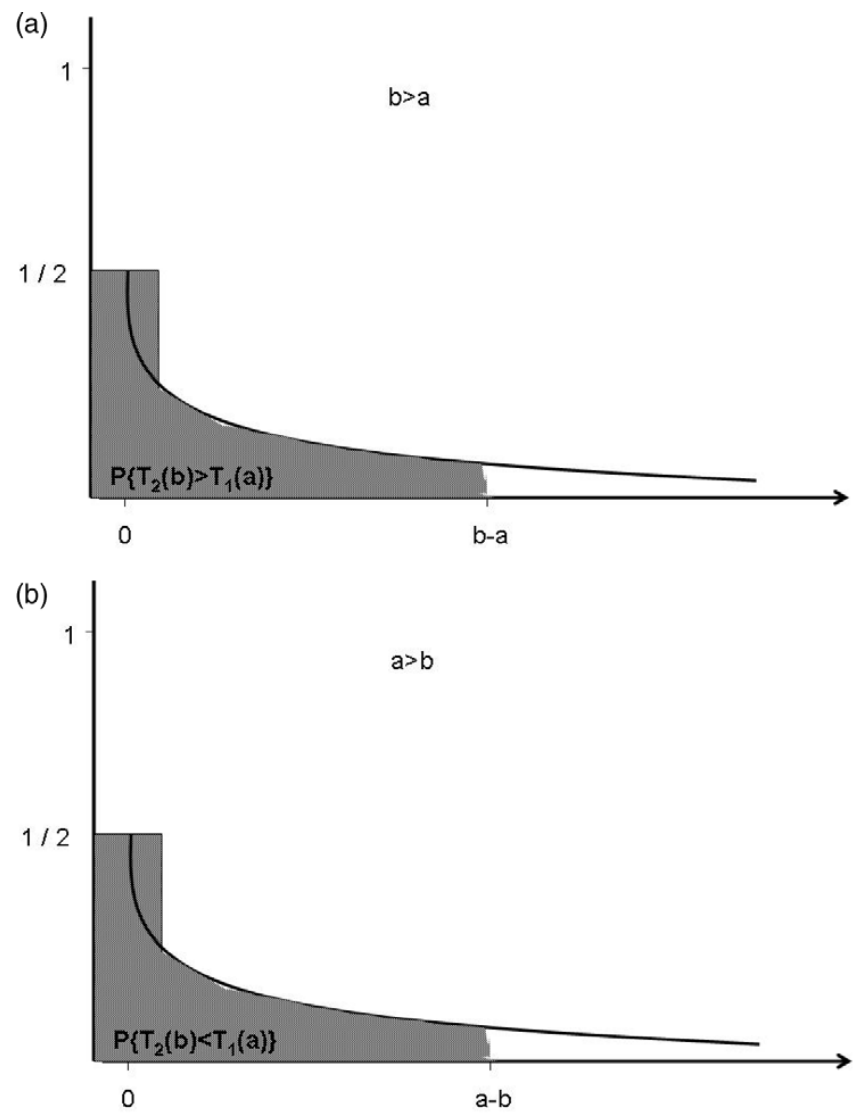

Figure 2. (a) Distribution of the random variable $y_{T_{1}(a)}^{+}$for $\gamma=0$, and the gray area marks $P\left(T(a, b)=T_{1}(a)\right)$, in the case where $b \geq a$. (b) Distribution of the random variable $y_{T_{2}(b)}^{-}$for $\gamma=0$, and the gray area marks $P\left(T(a, b)=T_{2}(b)\right)$, in the case where $a \geq b$.

Thus

$$
\begin{aligned}
P\left(U_{1}(\alpha)<U_{2}(\beta)\right)= & P\left(T_{1}\left(-\frac{1}{\sigma} \log (1-\alpha)\right)\right. \\
& \left.<T_{2}\left(\frac{1}{\sigma} \log (1+\beta)\right)\right),
\end{aligned}
$$

and we can apply Theorem 2.1 with the following parameters: $a=-(1 / \sigma) \log (1-\alpha), \quad b=(1 / \sigma) \log (1+\beta) \quad$ and $\gamma=(\mu / \sigma)-(\sigma / 2)$. The resulting probability is given by

$$
\begin{aligned}
& P\left(U_{1}(\alpha)<U_{2}(\beta)\right) \\
& =\frac{(1+\beta)^{-\left[2\left(\mu / \sigma^{2}\right)-1\right]}+\left[2\left(\mu / \sigma^{2}\right)-1\right] \cdot \log (1+\beta)-1}{(1+\beta)^{-\left[2\left(\mu / \sigma^{2}\right)-1\right]}+(1+\beta)^{\left[2\left(\mu / \sigma^{2}\right)-1\right]}-2} \\
& \quad \times[(1-\alpha)(1+\beta)]^{\left[2\left(\mu / \sigma^{2}\right)-1\right] /[1-(1+\beta)]^{\left[2\left(\mu / \sigma^{2}\right)-1\right]}},
\end{aligned}
$$

when $\alpha \geq \beta /(1+\beta)$, and

$$
\begin{aligned}
& P\left(U_{1}(\alpha)<U_{2}(\beta)\right) \\
& =1-\frac{(1-\alpha)^{-\left[2\left(\mu / \sigma^{2}\right)-1\right]}+\left[2\left(\mu / \sigma^{2}\right)-1\right] \cdot \log (1-\alpha)-1}{(1-\alpha)^{-\left[2\left(\mu / \sigma^{2}\right)-1\right]}+(1-\alpha)^{\left[2\left(\mu / \sigma^{2}\right)-1\right]}-2} \\
& \quad \times[(1-\alpha)(1+\beta)]^{\left[2\left(\mu / \sigma^{2}\right)-1\right] /[1-(1-\alpha)]^{\left[\left(\mu / \sigma^{2}\right)-1\right]}}
\end{aligned}
$$

when $\alpha \leq \beta /(1+\beta)$. In the case when $\mu=(1 / 2) \sigma^{2}$, the above formulae simplify to

$$
P\left(U_{1}(\alpha)<U_{2}(\beta)\right)=\frac{1}{2}[(1-\alpha)(1+\beta)]^{1 / \log (1+\beta)},
$$

when $\alpha \geq \beta /(1+\beta)$, and

$$
P\left(U_{1}(\alpha)<U_{2}(\beta)\right)=1-\frac{1}{2}[(1-\alpha)(1+\beta)]^{1 / \log (1-\alpha)},
$$

when $\alpha \leq \beta /(1+\beta)$.

\section{Concluding remarks}

In this paper, we are able to compute the probability that a drawdown of size $a$ occurs before an upward rally of size $b$ in the drifted Brownian motion model and use this result to compute the probability that a drop of a given percentage $\alpha$ from the running maximum of a stock occurs before a rise of a given percentage $\beta$ from the running minimum of a stock.

In the case of a more general model, such as, for example, a general Markovian or non-Markovian model, there are some useful formulae that do result from renewal considerations, but we might not be able to obtain closed form expressions as they appear in Theorem 2.1. To be more specific, let us assume that we are in the case that $b>a$. Then the following equation holds regardless of the underlying model dynamics:

$$
\begin{aligned}
P\left(T_{2}(b)<T_{1}(a)\right) & =P\left(T_{2}(a)<T_{1}(a)\right) \cdot P\left(T_{2}(b)\right. \\
& \left.<T_{1}(a) \mid T_{2}(a)<T_{1}(a)\right),
\end{aligned}
$$

where $P\left(T_{2}(a)<T_{1}(a)\right)$ can be readily computed using equation (37). This equation, in turn, is computed using equations (35) and (36), both of which follow by a renewal type of argument and hold regardless of the underlying model. However, the term $P\left(T_{2}(b)<\right.$ $\left.T_{1}(a) \mid T_{2}(a)<T_{1}(a)\right)$ will very clearly depend on the underlying model. This represents the probability that the rally process $y_{t}^{+}$will reach level $b$ before the drawdown process $y_{t}^{-}$reaches level $a$, given that $y_{t}^{+}$reached level $a$ before $y_{t}^{-}$did. That is the same as the probability that the underlying process travels an extra upward distance of $b-a$ before it drops below its running maximum by $a$ units. We can hope for a closed-form formula of this probability only in the case that the underlying process has independent increments. Notice that, in the case of independent increments, both the drawdown process and the rally process are Markovian. However, if the assumption of independent increments does not hold, the above conditional probability will be path dependent in even in a Markovian model, resulting in potentially complicated computations.

Similarly, it is important to notice that (27) is true regardless of the underlying model (the proof of this result is also based on renewal type of arguments and appears in the long equation before (29)). However, 
the exact distribution of $X_{T_{1}(a)}+a=\sup _{t \leq T_{1}(a)} X_{t}$ will depend heavily on the assumption of the underlying model. Our results can be applied in a straightforward way for diffusion models for which the distribution of $\sup _{t \leq T_{1}(a)} X_{t}$ is explicitly known, which is a fairly large class of diffusions (see, for instance, Lehoczky (1977)).

As a consequence of the fact that our results address diffusion models that satisfy the conditions that appear in Lehoczky (1977), important financial models of a mean-reverting character or drift-varying character with a constant adjustment coefficient are included. For instance, the Cox-Ingersoll-Ross model for interest rates falls into this setting. From that perspective our results might be used quite universally. Moreover, even in more general types of jump models that result in fattailed distributions, equations (57) and (37), as well as (27), all hold true, and could be used as starting points in the estimation of the probabilities of the events in Theorem 2.1 .

Finally, it is worth pointing out that, using these results, one can compute the expected time of the minimum of the stopping times $T_{1}(a)$ and $T_{2}(b)$. This is of great interest for detecting a regime change in the case of two-sided alternatives. Stopping times such as the minimum of $T_{1}(a)$ and $T_{2}(b)$ resemble the 2-CUSUM stopping rule that has traditionally been used in the detection of regime changes in the presence of two-sided alternatives (see, for example, Tartakovsky (1994) and Hadjiliadis and Moustakides (2005)).

\section{Acknowledgements}

The authors would like to thank Mark Brown, Chris Heyde and Steven Shreve for helpful comments on the manuscript. We are also thankful to the publishing editor David Burgoyne and the two anonymous referees for the suggested improvements to the paper. The work of Jan Večer was supported, in part, by National Science Foundation grant DMS 0418457.

\section{References}

Asmussen, S., Ruin Probabilities, 2000 (World Scientific: Singapore).

Beibel, M., A note on Ritov's Bayes approach to the minimax property of the CUSUM procedure. Ann. Statist., 1996, 24, 1804-1812.

Chekhlov, A., Uryasev, S. and Zabarankin, M., Drawdown measure in portfolio optimization. Int. J. Theor. Appl. Finan., 2005, 8, 13-58.

Hadjiliadis, O. and Moustakides, G.V., Optimal and asymptotically optimal CUSUM rules for change point detection in the Brownian motion model with multiple alternatives. Theor. Prob. Applic., 2005, 50, 131-144.

Lehoczky, J.P., Formulas for stopped diffusion processes with stopping times based on the maximum. Ann. Prob., 1977, 5, 601-607.

Magdon-Ismail, M., Atiya, A.F., Pratap, A. and Abu-Mostafa, Y.S., On the maximum drawdown of a Brownian motion. J. Appl. Prob., 2004, 41, 147-161.

Meijilson, I., The Time to a Given Drawdown in Brownian Motion, Vol. XXXVII of Seminaire de Probabilites, pp. 94-108, 2003 (Springer: Berlin).

Moustakides, G.V., Optimality of the CUSUM procedure in continuous time. Ann. Statist., 2004, 32, 302-315.

Page, E.S., Continuous inspection schemes. Biometrika, 1954, 41, 100-115.

Shiryaev, A.N., Minimax optimality of the method of cumulative sums (CUSUM) in the case of continuous time. Russ. Math. Surv., 1996, 51, 750-751.

Tartakovsky, A.G., Asymptotically minimax multi-alternative sequential rule for disorder detection, in Statistics and Control of Random Processes. Proceedings of the Steklov Institure of Mathematics, pp. 229-236, 1994 (American Mathematical Society: Providence RI).

Taylor, H.M., A stopped Brownian motion formula. Ann. Prob., 1975, 3, 234-246. 